\title{
Selection of Suitable Set of Decision Rules Using Choquet Integral
}

\author{
Laurent Wendling $^{1}$, Jan Rendek ${ }^{1}$, and Pascal Matsakis ${ }^{2}$ \\ ${ }^{1}$ LORIA - ESIAL, Universit Henri Poincar \\ 54506 Vandœuvre-ls-Nancy, France \\ ${ }^{2}$ CIS Dpt, Universit de Guelph \\ ON N1G 2W1 Canada \\ $\{$ wendling,rendek\}@loria.fr, matsakis@cis.uoguelph.ca
}

\begin{abstract}
An approach to automatically extract pertinent subsets of soft output classifiers, assumed to decision rules, is presented in this paper. They are aggregated into a global decision scheme using the Choquet integral. A selection scheme is defined that discards weak or redundant decision rules, keeping only the most relevant subset. An experimental study, based on real world data attest the interest of our method.
\end{abstract}

\section{Introduction}

A pattern recognition system can be roughly decomposed into three successive steps 6 18. Firstly, the shapes are extracted from their surrounding background. This step, called segmentation, relies heavily on a priori knowledge of the documents to be processed. Secondly, a representation is built from the extracted patterns. This representation allows to computationally judge the (dis)similarity between two patterns. It can either be a set of measurements performed on the patterns, forming a vector of features, or a symbolic description of how the pattern can be divided into basic shapes. Thirdly, a decision rule is built, using the representation of the pattern. This rule predicts to which class an observed pattern most likely belongs. It can either be built by introducing some expert knowledge about the patterns, or learned on a representative subset of labeled patterns. Surveys on pattern representation and classification techniques over the last decade fail to conclude whether a set of generic methods performing best on any kind of document, can be found 66311. Rather, a collection of techniques has been developed to address domain specific issues. For a given application, the choice of a pattern representation or a decision scheme relies at best on the extensive testing of several combinations of techniques. It is not uncommon that a designer chooses by making an educated guess between the techniques he has at hand, or between those he is the most acquainted with. A tempting approach is to combine several decision rules, based on various representations and classification schemes, instead of electing only one. The expected outcome is a more robust final decision, taking advantage of all the decision rules qualities. This approach appears well suited to cope with cases where the 
available data is too scarce to determine the best method by thorough testing, or to build robust decision rules by learning. Many classifier combination systems have been proposed and compared in the literature [9 16 1019]. In this paper, an improvement of the aggregation of decision rules using the Choquet integral is proposed. The Choquet integral is part of the aggregation techniques based on fuzzy integrals and have been successfully used as fusion operators in various applications.

\section{Background on Choquet Integral Fusion}

\subsection{Decision Rules Fusion}

Let us consider $m$ classes, $\mathcal{C}=\left\{C_{1}, \ldots, C_{m}\right\}$, and $n$ Decision Rules (DRs) $X=$ $\left\{D_{1}, \ldots, D_{n}\right\}$. When a new pattern $x^{o}$ is observed, we wish to find the class it most likely belongs to. Labeling this unknown pattern is a three-steps process. Firstly, for each decision rule $j$ and each class $i$, we compute $\phi_{j}^{i}$ the degree of confidence in the statement "According to $D_{j}, x^{o}$ belongs to the class $C_{i}$ ". Secondly, we combine all these partial confidence degrees into a global confidence degree by choosing a suitable aggregation operator $\mathcal{H}$. Thus, the global confidence degree in the statement " $x^{o}$ belongs to $C_{i}$ ", noted $\Phi\left(C_{i} \mid x^{o}\right)$, is given by:

$$
\Phi\left(C_{i} \mid x^{o}\right)=\mathcal{H}\left(\phi_{1}^{i}, \ldots, \phi_{n}^{i}\right)
$$

Finally, $x^{o}$ is assigned to the class for which the confidence degree is the highest.

$$
\operatorname{label}\left(x^{o}\right)=\underset{i=1}{\operatorname{mrg}} \max \Phi\left(C_{i} \mid x^{o}\right)
$$

Many aggregation operators were introduced in the literature. If the classification issue implies more than two classes, two learning approaches can be followed. Either each class $C_{i}$ is paired with his own aggregation operator $\mathcal{H}^{i}$, or a single global aggregation operator is learned. In the former case, the final decision is slightly modified as the global confidence degree depends on the operator associated with the class.

$$
\Phi\left(C_{i} \mid x^{o}\right)=\mathcal{H}^{i}\left(\phi_{1}^{i}, \ldots, \phi_{n}^{i}\right)
$$

\subsection{Fuzzy Measures and the Choquet Integral}

The Choquet integral was first introduced in the capacity theory [2 15]. Let us denote by $X=\left\{D_{1}, \ldots, D_{n}\right\}$ the set of $n$ decision rules, and $\mathcal{P}$ the power set of $\mathrm{X}$, i.e. the set of all subsets of $X$.

Definition 1. A fuzzy measure or capacity, $\mu$, defined on $X$ is a set function $\mu: \mathcal{P}(X) \longrightarrow[0,1]$, verifying the following axioms:

$\mu(\emptyset)=0, \mu(X)=1$

$A \subseteq B \Longrightarrow \mu(A) \leqslant \mu(B)$ 
Fuzzy measures generalize additive measures, by replacing the additivity axiom by a weaker one (monotonicity). Fuzzy measures embed particular cases including probability measure, possibility and necessity measures, or belief and plausibility functions. In our context of decision rules fusion, $\mu(A)$ represents the importance, or the degree of trust in the decision provided by the subset $A$ of DRs. The next step in building a final decision, is to combine the partial confidence degree according to each DR into a global confidence degree, taking those weights into account.

Definition 2. Let $\mu$ be a fuzzy measure on $X$. The discrete Choquet integral of $\phi=\left[\phi_{1}, \ldots, \phi_{n}\right]^{t}$ with respect to $\mu$, noted $\mathcal{C}_{\mu}(\phi)$, is defined by:

$$
\mathcal{C}_{\mu}(\phi)=\sum_{j=1}^{n} \phi_{(j)}\left[\mu\left(A_{(j)}\right)-\mu\left(A_{(j+1)}\right)\right]
$$

where (.) is a permutation and $A_{(j)}=\{(j), \ldots,(n)\}$ represents the $[j . . n]$ associated criteria in increasing order and $A_{(n+1)}=\emptyset$.

\subsection{Determining the Fuzzy Measure}

There are several methods to determine the most adequate fuzzy measure to be used for a given application and the most straightforward learning approach is based on optimization techniques. The aim is to find the fuzzy measure that minimizes best a criterion on the training set, such has the square error. Considering $\left(x^{k}, y^{k}\right), k=1, \ldots, l, l$ learning samples where $x^{k}=\left[x_{1}^{k}, \ldots, x_{n}^{k}\right]^{t}$ is a $\mathrm{n}$-dimensional vector, and $y^{k}$ the expected global evaluation of object $k$, the fuzzy measure can be determined by minimizing [5]: $E^{2}=\sum_{k=1}^{l}\left(\mathcal{C}_{\mu}\left(x_{1}^{k}, \ldots, x_{n}^{k}\right)-y^{k}\right)^{2}$. This criterion can be put under a quadratic program form and solved by the Lemke method. Nevertheless the method requires at least $n ! /[(n / 2) !]^{2}$ learning samples. When little data is available, matrices may be ill-conditioned, causing a bad behavior of the algorithm. To cope with the above problems, "heuristic" algorithms have been developed. To our knowledge, the algorithm providing the best approximation was proposed by Grabisch in [4]. It assumes that in the absence of any information, the most reasonable way to aggregate the partial matching degrees is to compute the arithmetic mean on all the inputs.

\subsection{Behavioral Analysis of the Aggregation}

The importance index is based on the definition proposed by Shapley in game theory [17. It is defined for a fuzzy measure $\mu$ and a rule $i$ as:

$$
\sigma(\mu, i)=\frac{1}{n} \sum_{t=0}^{n-1} \frac{1}{\left(\begin{array}{c}
n-1 \\
t
\end{array}\right)} \sum_{\substack{T \subseteq X \backslash i \\
|\bar{T}|=t}}[\mu(T \cup i)-\mu(T)]
$$


It can be interpreted as a average value of the marginal contribution $\mu(T \cup i)-$ $\mu(T)$ of the decision rule $i$ alone in all combinations. The interaction index [14] represents the degree of interaction between two decision rules. If the fuzzy measure is non-additive then some sources interact. The marginal interaction between $\mathrm{I}$ and $\mathrm{j}$, conditioned to the presence of elements of the combination $T \subseteq X \backslash i j$ is:

$$
\left(\Delta_{i j} \mu\right)(T)=\mu(T \cup i j)+\mu(T)-\mu(T \cup i)-\mu(T \cup j)
$$

Averaging this criterion over all the subsets of $T \subseteq X \backslash i j$ gives the interaction index of sources $\mathrm{i}$ and $\mathrm{j}$.

$$
I(\mu, i j)=\sum_{T \subseteq X \backslash i j} \frac{(n-t-2) ! t !}{(n-1) !}\left(\Delta_{i j} \mu\right)(T)
$$

A positive interaction index for two DRs $i$ and $j$ means that the importance of one DR is reinforced by the second. A negative interaction index indicates that the sources are antagonist, and their combined use impairs the final decision.

\section{Extraction of Decision Rules}

\subsection{Handling with Learning Error}

Lattices (associated to fuzzy measures) are initialized at the arithmetic mean, and are approximated using a training set via a gradient descent. From training pattern, $m$ training samples are created $\Phi^{1}, \ldots, \Phi^{m}$, with $\Phi^{i}=\left(\phi_{1}^{i}, \ldots, \phi_{m}^{i}\right)$ where $\phi_{j}^{i}$ represents the confidence in the fact that the sample belongs to class $i$, according to DR $j$. Each of these samples is paired with a target value, i.e. the value an ideal operator would output using this sample as input. For techniques that use a single fuzzy measure no real formula exists and often the following one is used:

$$
\mathcal{C}_{\mu}\left(\Phi^{i}\right)=\left\{\begin{array}{l}
1, \text { if sample belongs to class } i \\
0, \text { otherwise }
\end{array}\right.
$$

For techniques that use a different fuzzy measure per class, the optimal target value minimizing the quadratic error is known for two classes [4]. Best criterion is defined as follows.

$$
E^{2}=\sum_{i} \sum_{j}\left|\Psi\left(\Delta \Phi_{12}\left(X_{k}^{j}\right)\right)-1\right|^{2}
$$

with $X_{k}^{j}$ is the $k$ th training data of class $j, \Psi$ a sigmoid-type function and $\Delta \Phi_{12}$ is given by:

$$
\Delta \Phi_{12}=\Phi_{\mu_{1}}\left(C_{1} \mid X_{k}^{j}\right)-\Phi_{\mu_{2}}\left(C_{2} \mid X_{k}^{j}\right)
$$

The error used in the gradient descent algorithm is given by:

$$
\left.e=\Psi\left(\Delta \Phi_{12}\left(X_{k}^{j}\right)\right)-1\right)
$$


The criterion is modified as follows to process with N-classes:

$$
\Delta \Phi_{q r_{r \in X-\{q\}}}=\Phi_{\mu_{q}}\left(C_{q} \mid X_{k}^{j}\right)-\bigotimes_{\{r \in X-\{q\}\}} \Phi_{\mu_{r}}\left(C_{r} \mid X_{k}^{j}\right)
$$

with $q$ and $r$ two classes. Operator $\otimes$ can be a median, min... Here the max was kept to move away ambiguous classes and so to favor a discrimate behavior. Median is interesting to preserve acceptable results even if some sources may be contradictory. The sign of $\Delta \Phi_{q r_{r \in X-\{q\}}}$ is studied to set the error to be propagated in the lattice as follows.

$$
\operatorname{sign}\left(\Delta \Phi_{q r_{r \in X-\{q\}}}\right)=\left\{\begin{array}{l}
+e=1 . / f\left(\Delta \Phi_{q r_{r \in X-\{q\}}}\right) \\
-0, e=0 \text { for } q, 1 \text { for others. }
\end{array}\right.
$$

with $f$ an increasing function.

\subsection{Weaker Decision Rule Extraction}

Once the lattice is learned, the individual performance of each DR is analysed in the produced fuzzy measure. This analysis is performed using the importance and interaction indexes defined in 2.4. The aim is to track the DRs having the weak importance in the final decision, and that positively interact the least with the other rules. Such DRs are assuming to blur the final decision. First low significant rules $S_{L}$ having an importance index lower than 1 are selected:

$$
S_{L}=\{k \mid n \cdot \sigma(\mu, k)<1\}
$$

The set of rules to be removed $M S_{L}$ is composed of the rules having an interaction index lower than the mean of the interaction indexes of $S_{L}$ :

$$
M S_{L}=\left\{k \mid \sum_{j=1, n} I(\mu, k j)<m\right\}_{k \in S_{L}}
$$

with the global mean interaction index $m=\frac{1}{\left|S_{L}\right|} \sum_{k \in S_{L}} \sum_{j=1, n} I(\mu, k j)$

\subsection{Extracting Decision Rules}

We use the classic fuzzy pattern matching setup. Each class $C_{1}, \ldots, C_{n}$ is associated with a fuzzy measure $\mu_{1}, \ldots, \mu_{n}$. The evaluation measures are generally classifier dependant [13. For their computation the classification of training data must be performed to identify the fuzzy measure for each class. The application of our learning algorithm followed by the extraction of the most relevant DRs forms a training epoch is quite similar to global scheme but regarding a set of classes aggregated at las using a argmax criterion. As it is not easy to valuate each combinaison of classes. we consider them indepedently. A Greedy feature selection algorithm is used to ensure a continuous extraction of unexpected features per class. At each epoch the weakest descriptor calculated from indices 
is removed and so on, while improving a gain (recognition rates). The overall algorithm is described below.

$\%$ Initialization $\%$

For $\operatorname{Each} C_{i} \mathcal{C}$

- Learned $\mu_{i}$

- Extract weakest descriptor

\section{End For Each}

$\%$ Main \%

While Minimization is on the way

- Replace old capacity with new capacity

- Evaluate gain (minimizing cost function)

- Keep the "best" new capacity

- Extract feature for that new capacity

\section{End while}

\section{Experimental Results}

The proposed approach being aimed at situations where little information is available for training, databases having a fair number of categories and a small number of samples have been used. For the experiments, several decision rules are set from different photometric descriptors associated to a basic similarity measure to belong in the same range. A set of nine pattern recognition methods $R=\left\{R_{i}\right\}_{i=1,9}$ is used here, most of them having a low processing time, easy to implement, and invariant to affine transforms such as translation, rotation, or scaling. The descriptors computed on the shapes are: $D C=\{$ ART [8], angular signature [1], GFD [22], Ellipticity, $f_{0}$ and $f_{2}$-histograms [12], moments of Zernike [7, Yang [21], Radon signature 20] . B1(9 classesx11samples) and B2 $(18,12)$ are well-known sharvit' databases (see figure1).

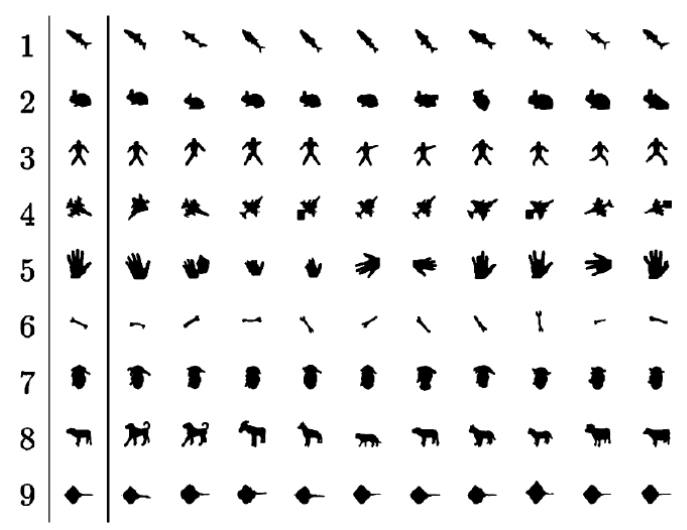

Fig. 1. The first Sharvit' database (B1) 
1

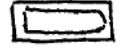

3<smiles>C1=CCC1</smiles>

4

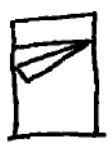

5
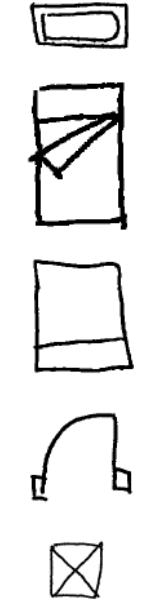

6<smiles>C1CC2CC3CC(C3)C(C1)C2</smiles><smiles>C1CCC2CCC(C1)C2</smiles>
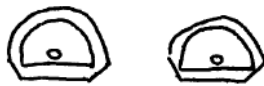

7

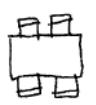

9
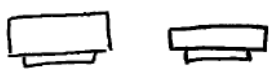

대

10

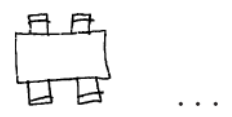

Fig. 2. Samples of the CVC database (B3)

Table 1. Recognition rates reached for each method

\begin{tabular}{|l|ccccccccc|ccc|c|}
\hline & $R_{1}$ & $R_{2}$ & $R_{3}$ & $R_{4}$ & $R_{5}$ & $R_{6}$ & $R_{7}$ & $R_{8}$ & $R_{9}$ & $M i$ & $M a$ & $M e$ & $C h$ \\
\hline B1 & 93 & 59 & 98 & 48 & 74 & 50 & 96 & 85 & 66 & 43 & 99 & 78 & 100 \\
\hline B2 & 88 & 56 & 90 & 48 & 62 & 46 & 86 & 66 & 56 & 59 & 87 & 76 & 93 \\
\hline B3 & 80 & 30 & 38 & 22 & 20 & 46 & 80 & 76 & 56 & 46 & 82 & 78 & 89 \\
\hline
\end{tabular}

B3(10x300) is a database kindly provided by the CVC Barcelone. Symbols have been drawn by ten people using "anoto" concept. Few samples are provided in figure 2 .

An experimental study is carried out from each decision rules $R_{i}$, basic aggregation operators as Min, Max, Median and the proposed method based on Choquet integral.

For each test, a crossvalidation was applied $(1 / 3$ and $2 / 3)$. Table 1 shows the good behavior of the method on these databases. The results reached independently by each decision rule $R_{i}$ are coherent with the amount of information processed by associated features. Simple aggregation operators: minimum, maximum and median exhibit various behavior depending on the database. In terms of recognition rates, only the maximum achieves better results than the best simple DR on two databases. In comparison to the simple DRs and the simple aggregation operators, our fusion operators based on the Choquet integral consistently achieve better results on each databases, in terms of recognition rates. On each dataset, our recognition method at worse improves a little the recognition rates; it never worsens them. Moreover a decreasing of the number of 
decision rules around $50 \%$ per class has been obtained. It allows to define a kind of identity map per class consisting in the most suitable decision rules following the application under consideration.

\section{Conclusion}

The algorithm appears well suited for the situation when no a priori knowledge is available about the relevance of a set of decision rules for a given dataset, or when the training set available is to small to build reliable decision rules, or to determine which method is the best. It finds the best consensus between the rules, taking their interaction into account, and discarding the undependable or redundant ones. A way to merge both numerical and expert decision rules in order to improve the recognition process is under consideration.

\section{References}

1. Bernier, T., Landry, J.-A.: A new method for representing and matching shapes of natural objects. Pattern Recognition 36(8), 1711-1723 (2003)

2. Choquet, G.: Theory of capacities. Annales de l'Institut Fourier 5, 131-295 (1953)

3. Cordella, L.P., Vento, M.: Symbol recognition in documents: a col of technics? International Journal of Document Analysis and Recognition 3(2), 73-88 (2000)

4. Grabisch, M.: A new algorithm for identifying fuzzy measures and its application to pattern recognition. In: Int. conf. FUZZ'IEEE 1995, pp. 145-150 (1995)

5. Grabisch, M., Nicolas, J.M.: Classification by fuzzy integral - performance and tests. Fuzzy Sets and Systems 65, 255-271 (1994)

6. Jain, A.K., Duin, R.P.W., Mao, J.: Statistical Pattern Recognition: A Review. IEEE Transactions on Pattern Analysis and Machine Intelligence 22(1), 4-37 (2000)

7. Khotanzad, A., Hong, Y.H.: Invariant Image Recognition by Zernike. IEEE Transactions on Pattern Analysis and Machine Intelligence 12(5), 489-497 (1990)

8. Kim, W.-Y., Kim, Y.-S.: A new region-based shape descriptor. In: TR 15-01, Pisa, Italy (1999)

9. Kittler, J., Hatef, M., Duin, R., Matas, J.: On combining classifiers. IEEE Transactions on Pattern Analysis and Machine Intelligence 20(3), 226-239 (1998)

10. Kuncheva, L.I., Whitaker, C.J.: Measures of diversity in classifier ensembles. Machine Learning 51, 181-207 (2003)

11. Lladós, J., Valveny, E., Sánchez, G., Martí, E.: Symbol Recognition: Current Advances and Perspectives. In: Blostein, D., Kwon, Y.-B. (eds.) GREC 2001. LNCS, vol. 2390, pp. 104-127. Springer, Heidelberg (2002)

12. Matsakis, P., Wendling, L.: A New Way to Represent the Relative Position Between Areal Objects. IEEE Transactions on Pattern Analysis and Machine Intelligence 21(7), 634-643 (1999)

13. Mikenina, L., Zimmermann, H.-J.: Improved feature selection and classification by the 2-additive fuzzy measure. Fuzzy Sets and Systems 107, 197-218 (1999)

14. Murofushi, T., Soneda, S.: Techniques for reading fuzzy measures(iii): interaction index. In: Proc. of the 9th Fuzzy Set System, pp. 693-696 (1993)

15. Murofushi, T., Sugeno, M.: A theory of fuzzy measures: representations, the Choquet integral, and null sets. Journal of Math. Anal. Appl. 159, 532-549 (1991) 
16. Ruta, D., Gabrys, B.: An overview of classifier fusion methods. Comp. and Information System 7(1-10) (2000)

17. Shapley, L.: A value for n-person games. In: Khun, H., Tucker, A. (eds.) Annals of Mathematics Studies, pp. 307-317. Princeton University Press, Princeton (1953)

18. Smeulders, A.W.M., Worring, M., Santini, S., Gupta, A., Jain, R.: CB Image Retrieval at the End of the Early Years. IEEE Transactions on Pattern Analysis and Machine Intelligence 22(12), 1349-1380 (2000)

19. Stejic, Z., Takama, Y., Hirota, K.: Mathematical aggregation operators in image retrieval: effect on retrieval performance and role in relevance feedback. Signal Processing 85(2), 297-324 (2005)

20. Tabbone, S., Wendling, L.: Binary shape normalization using the Radon transform. In: Nyström, I., Sanniti di Baja, G., Svensson, S. (eds.) DGCI 2003. LNCS, vol. 2886, pp. 184-193. Springer, Heidelberg (2003)

21. Yang, S.: Symbol Recognition via Statistical Integration of Pixel-Level Constraint Histograms: A New Descriptor. IEEE Transactions on Pattern Analysis and Machine Intelligence 27(2), 278-281 (2005)

22. Zhang, D., Lu, G.: Shape-based image retrieval using generic fourier descriptor. Signal Processing: Image Communication 17, 825-848 (2002) 